\title{
Indentation of Single-Crystal Silicon Nanolines: Buckling and Contact Friction at Nanoscales"
}

Bin Li, ${ }^{1}$ Qiu Zhao, ${ }^{1}$ Huai Huang, ${ }^{1}$ Zhiquan Luo, ${ }^{1}$ Min K. Kang, ${ }^{2}$ Jang-Hi Im, ${ }^{1}$

Richard A. Allen, ${ }^{3}$ Michael W. Cresswell, ${ }^{3}$ Rui Huang, ${ }^{2}$ and Paul S. $\mathrm{Ho}^{1, \mathrm{a})}$

${ }^{1}$ Microelectronics Research Center, The University of Texas at Austin, 10100

Burnet Road, Bldg 160, Austin, Texas 78758, USA

${ }^{2}$ Department of Aerospace Engineering and Engineering Mechanics, University of

Texas, Austin, Texas 78712, USA

${ }^{3}$ Semiconductor Electronics Division, National Institute of Standards and

Technology, Gaithersburg, Maryland 20899, USA

Keywords: Silicon nanolines, Anisotropic wet etching, Nanoindentation

\begin{abstract}
:
High-quality single-crystal silicon nanolines (SiNLs) with $24 \mathrm{~nm}$ line width and a height/width aspect ratio of 15 were fabricated. The mechanical properties of the SiNLs were characterized by nanoindentation tests with an atomic force microscope (AFM). The indentation load-displacement curves showed an instability with large displacement bursts at a critical load ranging from $9 \mu \mathrm{N}$ to $30 \mu \mathrm{N}$. This phenomenon was attributed to a transition of the buckling mode of the SiNLs under indentation,

ฯ Contribution of the National Institute of Standards and Technology (NIST). Not subject to copyright.
\end{abstract}


which occurred preceeding the final fracture of the nanolines. The mechanics of SiNLs under indentation was analyzed by finite element simulations, which revealed two different buckling modes depending on the contact friction at the nanoscale.

a) Electronic mail: paulho@mail.utexas.edu 


\section{INTRODUCTION}

Silicon nanostructures are essential building blocks for nanoelectronic devices and nano-electromechanical systems (NEMS). ${ }^{[1-4]}$ Mechanical characterization at nanoscales is important for practical applications but remains challenging as the mechanical properties such as yield strength, fracture strength, contact and friction properties, are often different at the nanoscale from their bulk properties. In this work, single-crystal silicon nanolines (SiNLs) were characterized by nanoindentation tests using an atomic force microscope (AFM).

Various processes have been developed for fabrication of silicon nanostructures. ${ }^{[5-12]}$ By combining electron-beam lithography (EBL) and anisotropic wet etching (AWE), a top-down fabrication process was developed to obtain singlecrystal, parallel SiNLs on a silicon (110) wafer. $^{[5]}$ Under indentation, the singlecrystal SiNLs showed mechanical properties that are distinctly different from those of bulk silicon. In a previous study of SiNLs with a line width of $74 \mathrm{~nm}$ and an aspect ratio of 6.9, the load-displacement curves from the indentation tests showed an instability with large displacement bursts at a critical load ranging from $480 \mu \mathrm{N}$ to $700 \mu \mathrm{N} .^{[5]}$ This phenomenon was attributed to a transition of the buckling mode of the SiNLs under indentation, which occurred preceeding the final fracture of the nanolines. In our earlier work, the onset of buckling instability and the mode transition were found to depend on the friction at the contact between the indenter tip and the SiNLs as well as the relative tip location and the aspect ratio of the SiNLs ${ }^{[13]}$ However, due to the close spacing of the SiNLs, we were not able to control the tip location precisely with respect to any particular nanoline, and consequently the contact friction was not quantified. The present study improves the 
fabrication process to yield SiNLs with narrower line widths and wider line spacing to allow precise positioning of the indenter. The unique configuration and structural perfection of the single-crystal SiNLs made it possible to quantitatively investigate the buckling phenomenon and contact friction at the nanoscale.

\section{FABRICATION OF SILICON NANOLINES}

A low-pressure chemical vapor deposition (LPCVD) process was used to grow 20 nm thick oxide on a Si (110) wafer. E-beam evaporation was then employed to deposit a $\sim 15 \mathrm{~nm}$ thick Cr layer over the oxide. Patterns of parallel SiNLs were formed with an e-beam exposure system operating at $50 \mathrm{KV}$ on a spin coated e-beam resist of thickness $\sim 130 \mathrm{~nm}$. Pattern transfer from the resist to the $\mathrm{Cr}$ layer was performed by a $\mathrm{Cl}_{2}$ and $\mathrm{O}_{2}$ plasma at a pressure of $80 \times 10^{-3} \mathrm{~Pa}$ and $\mathrm{RF}$ power of $75 \mathrm{~W}$ for $2 \mathrm{~min}$. The exposed oxide layer was etched in a $\mathrm{CHF}_{3}$ and $\mathrm{O}_{2}$ plasma down to the (110) silicon surface using the Cr lines as the etching mask. Subsequently, the residual resist was removed in a Piranha solution, and TMAH heated to $80^{\circ} \mathrm{C}$ was used to etch silicon along the (111) planes of silicon. Finally, the Cr and oxide layers were removed by Transene Chromium Etching Solution 1020 and buffered oxide-etching solution, respectively.

The high-resolution EBL method is capable of producing patterns with feature dimensions less than $20 \mathrm{~nm}$. Combining with high-quality pattern transfer by AWE, the process yielded SiNLs with straight and nearly atomically flat sidewalls without defects induced by reactive ion or plasma etching. In this study, SiNLs with line widths as small as $24 \mathrm{~nm}$ and a height-to-width aspect ratio exceeding 15 have 
been fabricated. Fig. 1 shows two SEM images of one set of the fabricated SiNLs. The line width and the height of these SiNLs were about $24 \mathrm{~nm}$ and $380 \mathrm{~nm}$, respectively. The trench width was $366 \mathrm{~nm}$, and the line length was about $30 \mu \mathrm{m}$. These SiNLs have smooth sidewalls, almost perfectly rectangular cross sections, and a highly uniform line width. The line-width and spacing of the SiNLs was chosen to allow the indenter tip to be precisely positioned at the center of the trench between adjacent lines to allow quantification of the contact configuration and friction.

\section{NANOINDENTATION TESTS}

An AFM based nanoindentation system (a Triboscope system from Hysitron, Inc. ${ }^{1}$ was employed to measure the mechanical properties of the SiNLs, using a conically shaped indenter with the tip radius around $3.5 \mu \mathrm{m}$. Before each indentation test, the indenter tip was placed directly above a $30 \mu \mathrm{m}$ by $30 \mu \mathrm{m}$ grating area patterned with an array of parallel nanolines. For a better control over the indentation position, the indenter was manipulated to position precisely on top of a pattern trench center, guided by an AFM image of the SiNLs using the same conical indenter in the image-scanning mode. Subsequently, the indenter was slowly brought into contact with the SiNLs, and load-controlled indentation tests were performed with a dwell time of 1 second at the peak of a trapezoidal loading profile.

Figure 2 shows two sets of force-displacement curves obtained from the nanoindentation tests. Similar to the indentation response of the $74 \mathrm{~nm}$ wide SiNLs

\footnotetext{
${ }^{1}$ Certain commercial equipment, instruments, or materials are identified in this document in order to specify adequate measurement procedures. Such identification does not imply recommendation or endorsement by the National Institute of Standards and Technology, nor does it imply that the materials or equipment identified are necessarily the best available for the purpose.
} 
reported in our previous work, ${ }^{[5]}$ a large displacement burst was observed at a critical indentation load, which was attributed to buckling instability of the SiNLs. However, two distinct deformation modes were observed from the present data for the high-aspect-ratio $24 \mathrm{~nm}$ SiNLs, termed as mode A (Fig. 2a) and mode B (Fig. 2b), respectively. As shown in Fig. 2a for the deformation mode A, the critical load for the displacement burst ranged from $9 \mu \mathrm{N}$ to $17 \mu \mathrm{N}$. Beyond the critical load, stair-like increases of the indentation load were observed and the displacement went up to $\sim 220 \mathrm{~nm}$ under a $\sim 70 \mu \mathrm{N}$ force. For the deformation mode B (Fig. 2b), however, the critical loads are noticeably higher, ranging from $24 \mu \mathrm{N}$ to $30 \mu \mathrm{N}$. On the other hand, the displacement under the same $70 \mu \mathrm{N}$ force was only $\sim 80 \mathrm{~nm}$, much smaller than that in mode A. It appears that the SiNLs in mode B had a much higher indentation stiffness than those in mode A, although the indentation loaddisplacement curves before the critical load were essentially identical. Since the indentation tests were performed on the same array of SiNLs at different locations, the two different deformation modes were most likely due to the variation of local contact properties as a result of surface irregularities of the spherical tip and SiNLs. Our previous study on the $74 \mathrm{~nm}$ SiNLs suggested that, even for the same buckling mode, the critical load depends on the local contact friction between the indenter and the SiNLs. ${ }^{[5,13]}$ It is found from the present study that, for the high-aspect-ratio 24 $\mathrm{nm}$ SiNLs, variation in the local friction property can lead to two drastically different buckling modes (see Fig. 3).

Owing to their narrow linewidth and high aspect ratio, the critical indentation loads for the $24 \mathrm{~nm}$ SiNLs are much lower than those of the $74 \mathrm{~nm}$ lines reported in 
the previous work. ${ }^{[5]}$ On the other hand, the maximum indentation displacement for the $24 \mathrm{~nm}$ SiNLs was larger, up to $220 \mathrm{~nm}$. This displacement is about 58\% of the line height. Remarkably, as shown in both Fig. 2a and Fig. 2b, the significant displacements appeared to be fully elastic, with no residual deformation observed after withdrawal of the indenter for multiple experiments. Furthermore, the unloading paths in each set of the indentation tests coincided well with each other, indicating a good repeatability in the experiments. As will be shown later, the large recoverable deformation of the SiNLs was a result of significant bending as the vertically aligned nanolines buckled elastically under indentation.

\section{MODELING AND DISCUSSIONS}

A finite element model (FEM) was developed to simulate the indentation

tests. The model was similar to that described in our previous work. ${ }^{[5,13]}$ Since the indenter was located precisely on the trench center in the indentation tests, the relative tip location was fixed in the model, while the friction coefficient at the contact between the indenter surface and the SiNLs was varied. A simple Coulomb friction model was adopted to study the effect of the frictional contact. The indenter was modeled as a rigid body with a spherical surface of radius $3.5 \mu \mathrm{m}$. In each simulation, a vertical displacement was applied to a reference node of the indenter, and the indentation force was determined from the reaction force at the reference node. Since the indentation deformation was typically localized within a region much smaller than the area of the SiNL gratings, the boundary conditions at the ends of the nanolines have negligible effect as long as the tip of the indenter was located more than about $5 \mu \mathrm{m}$ away from the boundaries. In the model, the line length was 
set to be $6 \mu \mathrm{m}$, which was deemed sufficient to simulate the local deformation of the long lines. The elastic properties of bulk silicon were used for the SiNLs, which was justified by the close agreement in the load-displacement curves (up to the critical load) between the modeling results and the measurements. The agreement also suggested that the elastic properties of Si effectively remain size-independent with the line width to $24 \mathrm{~nm}$.

Figures $3 a$ and $3 b$ show the simulated deformation of SiNLs under indentation with two different friction coefficients at the contact. With a relatively low friction coefficient ( $\mu=0.04$ ), the SiNLs buckled outwards with significant sliding along the indenter surface. A transition from predominantly compression to bending deformation of the SiNLs leads to the observed displacement burst at a critical load, similar to the buckling instability of a compressed column. When the friction coefficient is higher ( $\mu=0.06$ in Fig. 3b), however, the frictional force at the contact surface prevents the two innermost SiNLs from sliding outwards, leading to a different buckling mode. The friction coefficient required to prevent sliding depends on the contact angle. A simple analysis (see Appendix) predicts that the critical friction coefficient is: $\mu_{c}=S / 2 R$, where $S$ is the trench width of the SiNLs and $R$ the radius of the indenter tip. This corresponds to a value of $\mu_{c}=0.052$ for the SiNLs in the present study, in agreement with the FEM numerical simulations. In Fig. 3b, the outer two SiNLs slid outwards because an even larger frictional coefficient $(\mu>0.11)$ is required to prevent sliding of these lines further away from the tip. Figure 4 plots the critical loads obtained from the numerical simulations 
versus the local friction coefficient, clearly showing the transition from the first buckling mode $\left(\mu<\mu_{c}\right)$ to the second buckling mode $\left(\mu>\mu_{c}\right)$.

Figure 5 shows the comparisons between the indentation force-displacement curves from the numerical simulations with low friction coefficients $(\mu<0.05)$ and the experimental data corresponding to the deformation mode A. The effect of friction coefficient in this regime is illustrated in Fig. 5a. All the simulated forcedisplacement curves coincided in the initial loading part, where the deformation of the SiNLs was predominantly compressive and thus insensitive to the friction at the contact. For each simulation, a critical load is identified, beyond which the indentation force dropped as the displacement keep increasing until more lines were brought into contact. This predicts a sudden burst in displacement (horizontal arrows in Figs. 5b-d) at the critical load under a load-control experiment. The calculated critical load increased from $7 \mu \mathrm{N}$ to $29 \mu \mathrm{N}$ as the friction coefficient $\mu$ increased from 0 (frictionless) to 0.05 . Therefore, as the contact friction increases, the barrier increases for the onset of sliding and thus buckling of the SiNLs. It is noted that in the experimental data of Fig. 2, the force dropped slightly after the critical load as the displacement burst occurred. This phenomenon was due to the fact that the feedback control used in the indentation system was an open-loop type and thus the indentation force could not be fully controlled. To achieve better control of the loading profile, upgrading of the feedback control to a close-loop is in progress for future studies.

In Fig. 5b, the simulated indentation curve with the friction coefficient $\mu=$ 0.02 predicted a critical buckling load of $10 \mu \mathrm{N}$ and a displacement burst of $\sim 25 \mathrm{~nm}$ 
(from A to B), which compare well with two sets of experimental data. In Figs. 5c and 5d, numerical simulations with $\mu=0.03$ and 0.04 are compared to the other three data sets in the deformation mode A. The results indicate that both the critical load and the displacement burst increase with the friction coefficient depending on the local surface condition over the area of patterned SiNLs. The sensitivity of the critical load on the friction coefficient thus offers a potential approach for probing the local surface properties at nanoscale. Through these comparisons, the friction coefficients were estimated to be around $0.02-0.04$ for the experiments in the deformation mode A. These friction coefficients are larger than that in a previous work for SiNLs with $74 \mathrm{~nm}$ line width. ${ }^{[5]}$ The difference likely comes from the different contact conditions, including different indenter tips used in the tests and the different line dimensions.

Figure 6a shows four sets of simulated load-displacement curves with friction coefficient $\mu$ ranging from 0.04 to 0.1 . As the friction coefficient increased from 0.04 to 0.06 , the critical load increased significantly from $16 \mu \mathrm{N}$ to $55 \mu \mathrm{N}$, due to the change of the buckling mode as illustrated in Fig. 3. Within this range, the indentation response of the SiNLs (e.g., the critical load and the magnitude of displacement burst) is very sensitive to the friction coefficient. Further increasing the friction coefficient from 0.06 to 0.1 , the simulations showed little change in the indentation curves. Once the frictional force is sufficient to prevent sliding of the SiNLs, the critical load and the postbuckling behavior become insensitive to the friction coefficient. 
Figure 6b compares the simulated load-displacement curves with friction coefficient $\mu=0.05$ and 0.06 to the experimental data of the deformation mode B (Fig. 2b). It can be seen that the FEM results matched reasonably well with the experimental data up to the critical load. The critical loads from the three indentation tests were in a range of $24 \mu \mathrm{N}$ to $30 \mu \mathrm{N}$, close to the simulation with $\mu=0.05$. However, the magnitude of displacement bursts in the experiments was much smaller and the post-buckling response laid in between of the two simulation curves. One possible scenario for this behavior is: while the initial friction coefficient at the contact was around 0.05 and thus led to the observed critical load, a small increase in the friction coefficient occurred as the SiNLs slid along the indenter surface, and the change of the surface conditions at the contact prevented further sliding of the SiNLs. The non-uniform contact surfaces and the sensitivity of the buckling behavior for the friction coefficients between 0.05 and 0.06 could result in a hybrid buckling mode as sketched in Fig. 6c, different from the two buckling modes (Fig. 3a and 3b) obtained from the numerical simulations with uniform friction coefficients.

\section{FRACTURE OF SiNLS}

While Fig. 2 shows recoverable deformation of the SiNLs after remarkably large indentation displacements, further increasing of the peak load of the indentation tests led to irrecoverable residual deformation after unloading. Two representative load-displacement curves are shown in Fig. 7a for such indentation tests, with the corresponding SEM images in Figs. 6b and 6c. Clearly, fracture of the SiNLs occurred in these tests. The two indentation curves in Fig. 6a represent two 
different fracture behaviors of the SiNLs, correlating with the two deformation modes observed in Fig. 2. Test 1 had a relatively low critical load for the initial displacement burst, similar to the deformation mode A. Test 2 had a critical load similar to the deformation mode B. While the maximum indentation displacement in Test $2(\sim 270 \mathrm{~nm})$ was smaller than that of Test $1(\sim 320 \mathrm{~nm})$, it is interesting to note that the SiNLs in Test 2 were crushed into a large number of small fragments (Fig. 7c), while in Test 1 much less fragments were observed (Fig. 7b). It is thus conceived that the different buckling modes of the SiNLs as shown in Fig. 3 lead to different fracture behavior. For the first buckling mode (Fig. 3a), the maximum tensile stress occurs at the root of the two innermost SiNLs. As the maximum tensile stress reaches the fracture strength, cracks nucleate and propagate, resulting in relatively large fragments. For the second buckling mode (Fig. 3b), the maximum tensile stress could occur at multiple locations, as indicted by the arrows. Consequently, nucleation of multiple cracks may occur simultaneously and lead to a large number of small fragments. Therefore, the friction property at the contact not only determines the buckling mode (thus the critical load and the displacement burst) but also leads to different fracture behaviors of the SiNLs.

Comparing the force-displacement curves in Fig. 2a to Test 1 in Fig. 6a, it is conjectured that fracture of the SiNLs occurred at a critical indentation displacement between $220 \mathrm{~nm}$ and $250 \mathrm{~nm}$, under the deformation mode A. The critical strain to fracture is thus estimated to be $7.5-9.7 \%$, based on the maximum principal strain in the SiNLs calculated from the FEM simulations under the same indentation displacements. The deformation of the SiNLs under an indentation displacement of $220 \mathrm{~nm}$ is shown in Fig. 3a. We note that the estimated strain to failure is much 
higher than that for bulk silicon $(\sim 1 \%),{ }^{[14]}$ but similar fracture strains have been reported for micron to nanoscale Si beams and nanowires. ${ }^{[5,15,16]}$ More systematic experimental data are needed for statistical analysis of the fracture phenomena to further understand the scale effect on fracture of silicon.

\section{CONCLUSIONS}

The present study demonstrated a top-down process combining EBL and AWE techniques to fabricate high-quality SiNLs with the line width as small as 24 $\mathrm{nm}$ and the height-to-width aspect ratio above 15. SiNLs fabricated by the present approach without reactive-ion or plasma-etching-induced damage in the pattern transfer process had nearly atomically smooth sidewalls, perfectly rectangular cross sections, and a highly uniform line width. The nanoindentation technique along with FEM simulations was used to quantitatively characterize the buckling and contact friction of the SiNLs. The geometry and the dimension of the SiNLs enabled us to precisely position the indentor tip at the trench center between two adjacent lines so that the contact configuration and friction can be quantified. The friction at the contact between the tip of the indenter and the SiNLs was found to play a critical role in determining the buckling modes and fracture mechanisms of the SiNLs. Friction coefficients in the range of $0.02 \sim 0.05$ were deduced from the present study at the nanoscale. These are much smaller than those reported at larger scales (> 0.1). ${ }^{[17,18]}$ This interesting result of the scaling effect on friction may be due to highly localized nature of the nanoscale contact and shall be further investigated. 


\section{ACKNOWLEDGEMENTS}

The authors thank Dr. Li Shi for helpful discussions on the fabrication process development. The work was performed in part at the Microelectronics Research Center of the University of Texas at Austin, a member of the National Nanofabrication Infrastructure Network supported by the National Science Foundation under Grant No. 0335765. MKK and RH are grateful for the financial support by the National Science Foundation through Grant No. CMMI-0654105. We are also grateful to the NIST Office of Microelectronics Programs for supporting this work.

\section{APPENDIX: Estimation of the critical friction coefficient}

As shown schematically in Fig. 8, the contact forces acting on the nanoline include a normal force $N$ and a friction force $f$. By the Coulomb friction model, the friction force is proportional to the normal force, i.e., $f=\mu N$, where $\mu$ is the friction coefficient at the contact. The normal force has a horizontal component that pushes the nanoline to slide away from the tip, while the horizontal component of the friction force opposes sliding. The competition defines a critical condition when the two horizontal components are balanced, i.e., $N \sin \alpha=f \cos \alpha$, where $\alpha$ is the contact angle between the normal direction of the indenter surface and the vertical nanoline. Consequently, the critical friction coefficient is: $\mu_{c}=\tan \alpha$. For the present study, since the tip radius is much larger than the line width and line spacing, the contact angle $\alpha$ is small and approximately, $\alpha \approx S / 2 R$, where $S$ is the trench width and $R$ the tip radius of the indenter. Thus, we have $\mu_{c} \approx S / 2 R$. 


\section{References:}

${ }^{1}$ C. M. Lieber, Z. L. Wang, MRS Bulletin 32, 99 (2007).

${ }^{2}$ F. Patolsky, G. Zheng, C. M. Lieber, Nanomedicine 51, 51 (2006).

${ }^{3}$ Y. Xia, P. Yang, Y. Sun, Y. Wu, B. Mayers, B. Gates, Y. Yin, F. Kim, H. Yan, Adv. Mater. 5, 353 (2003).

${ }^{4}$ J. -Y. Yu, S. -W. Chung, and J. R. Heath, J. Phys. Chem. B 104, 11864 ( 2000).

${ }^{5}$ B. Li, M. K. Kang, G. Lu, R. Huang, P. S. Ho, R. Allen, M. W. Cresswell, Nano Lett. 8, 92 (2008).

${ }^{6}$ J. Hu, T. W. Odom, C. M. Lieber, Acc. Chem. Res. 32, 435(1999).

${ }^{7}$ S. -W. Chung, J. -Y, J. R. Heath, Appl. Phys. Lett. 76, 2068 (2000).

${ }^{8}$ Y. Wu, P. Yang, J. Am. Chem. Soc. 123, 3165 (2001).

${ }^{9}$ M. Tabib-Azar, M. Nassirou, R. Wang, S. Sharma, T. I. Kamins, M. S. Islam, R. S. Williams, Appl. Phys. Lett. 87, 113102 (2005).

${ }^{10}$ M. Saif Islam, S. Sharma, T. I. Kamins, R. S. Williams, Appl. Phys. A 80, 1133 (2005).

${ }^{11}$ N. A. Melosh, A. Boukai, F. Diana, B. Gerardot, A. Badolato, P. M. Petroff, J. R. Heath, Science 300, 112 (2003).

${ }^{12}$ R. A. Beckman, E. Johnston-Halperin, N. A. Melosh, Y. Luo, J. E. Green, J. R. Heath, J. Appl. Phys. 96, 5921 (2004).

${ }^{13}$ M. K. Kang, B. Li, P.S. Ho, R. Huang, Journal of Nanomaterials 2008, 132728 (2008).

${ }^{14}$ R. F. Cook, J. Mat. Sci., 41, 841 (2006).

${ }^{15}$ T. Namazu, Y. Isono, T. Tanaka, J. Microelectromechanical Systems 11, 125 (2002). 
${ }^{16}$ S. Hoffmann, I. Utke, B. Moser, J. Michler, S. H. Christiansen, V. Schmidt, S. Senz, P. Werner, U. G ösele, C. Ballif, Nano Lett. 6, 622 (2006).

${ }^{17}$ B. Bhushan, X. Li, J. Mater. Res. 12, 54 (1997).

${ }^{18}$ D. Xu, K. Ravi-Chandar, K. M. Liechti, J. Colloid Interface Sci. 315, 772 (2007). 


\section{Figure Captions}

Fig. 1. SEM images of the parallel silicon nanolines, with $24 \mathrm{~nm}$ line width and 380 nm height. The line pitch is $390 \mathrm{~nm}$. (a) Plan view; the bright lines are SiNLs. (b) Cross-sectional view with $60^{\circ}$ tilt angle. A small trench pattern is specially designed at one end of the line to facilitate the cross-sectional SEM imaging, showing the sharp edges due to the anisotropic etching.

Fig.2. Load vs. displacement curves from the nanoindentation tests of the $24 \mathrm{~nm}$ SiNLs. (a) Deformation mode A, and (b) Deformation mode B. The SiNLs appeared to be stiffer than those in (a), with higher critical loads for the first displacement burst and smaller indentation displacements under same indentation forces.

Fig 3. Finite element simulations of the $24 \mathrm{~nm}$ SiNLs under indenation. (a) Deformation mode A: The nanolines buckled with significant sliding at the contact with a low friction coefficient, $\mu<0.05$. The indentation displacement was $220 \mathrm{~nm}$. (b) Deformation mode B: The two center lines buckled with no sliding at the contact point for a higher friction coefficient $(\mu=0.06)$. The indentation displacement was $60 \mathrm{~nm}$ in this case. The red arrows indicate the locations of the maximum tensile stresses that may eventually cause fracture.

Fig. 4. The critical indentation load predicted by the FEM model, as a function of the friction coefficient between the indenter and the SiNLs.

Fig. 5. Indentation force-displacement curves from finite element simulations of the $24 \mathrm{~nm}$ SiNLs. (a) Variation of the friction coefficient $\mu$ from 0 to 0.05 , showing increase of the critical load as $\mu$ increased. (b), (c) and (d): comparisons between the 
simulated load-displacement curves and the experimental data. The displacement bursts predicted by the FEM simulations are indicated by the horizontal arrows.

Fig. 6. (a) Simulated load vs. displacement curves of the $24 \mathrm{~nm}$ SiNLs under indentation with friction coefficient $\mu$ varying from 0.04 to 0.10 . (b) Comparison between the experimental data of deformation mode B (Fig. 2b) and the simulated load-displacement curves with friction coefficient $\mu=0.05$ and 0.06 . The magnitude of the displacement burst in each case was indicated by the horizontal arrows. (c) A schematic illustration of a hybrid buckling mode, with the top trapped after a partial sliding, possibly due to non-uniform friction properties at the contact surfaces.

Fig. 7. Further increase of the indentation load far beyond the critical load resulted in fracture of SiNLs, with large residual displacements observed after unloading of the indenter. (a) Load-displacement curves from two indentation tests, which showed the characteristic of deformation modes A and B, respectively. (b) and (c) are two SEM images of the fractured SiNLs after Test 1 and Test 2, respectively.

Fig. 8. Schematic of the contact geometry between the indenter and a nanoline, with the normal force $N$ and the friction force $f$ acting on the top of a nanoline. 

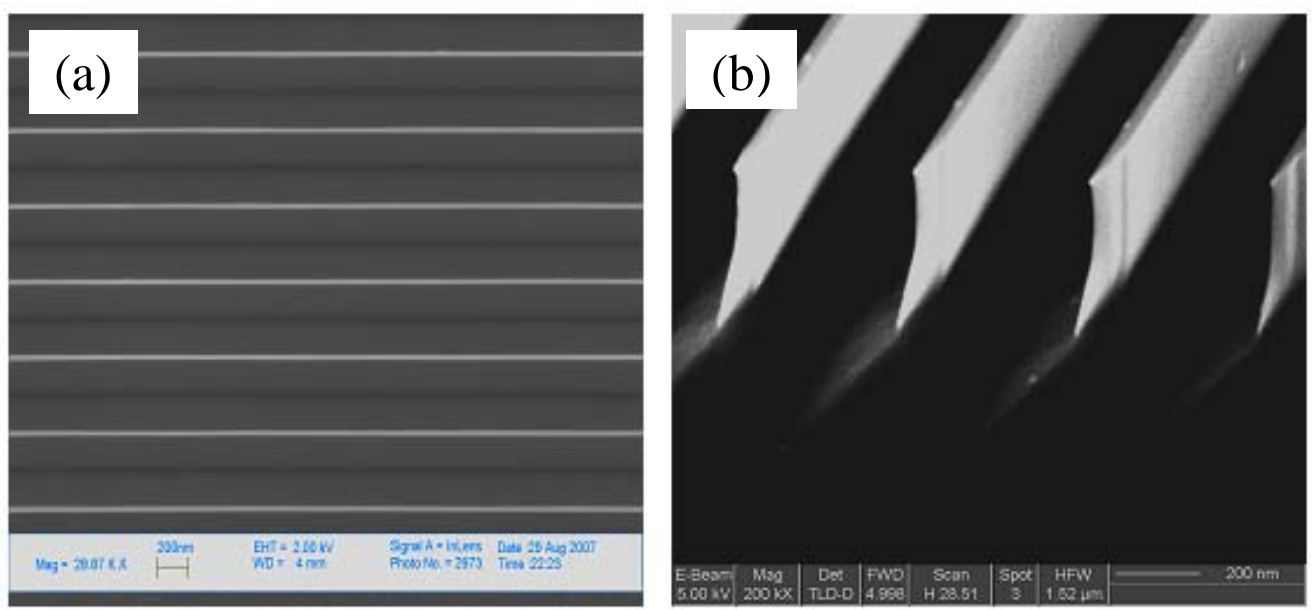

Fig. 1. SEM images of the parallel silicon nanolines, with $24 \mathrm{~nm}$ line width and 380 nm height. The line pitch is $390 \mathrm{~nm}$. (a) Plan view; the bright lines are SiNLs. (b) Cross-sectional view with $60^{\circ}$ tilt angle. A small trench pattern is specially designed at one end of the line to facilitate the cross-sectional SEM imaging, showing the sharp edges due to anisotropic etching. 

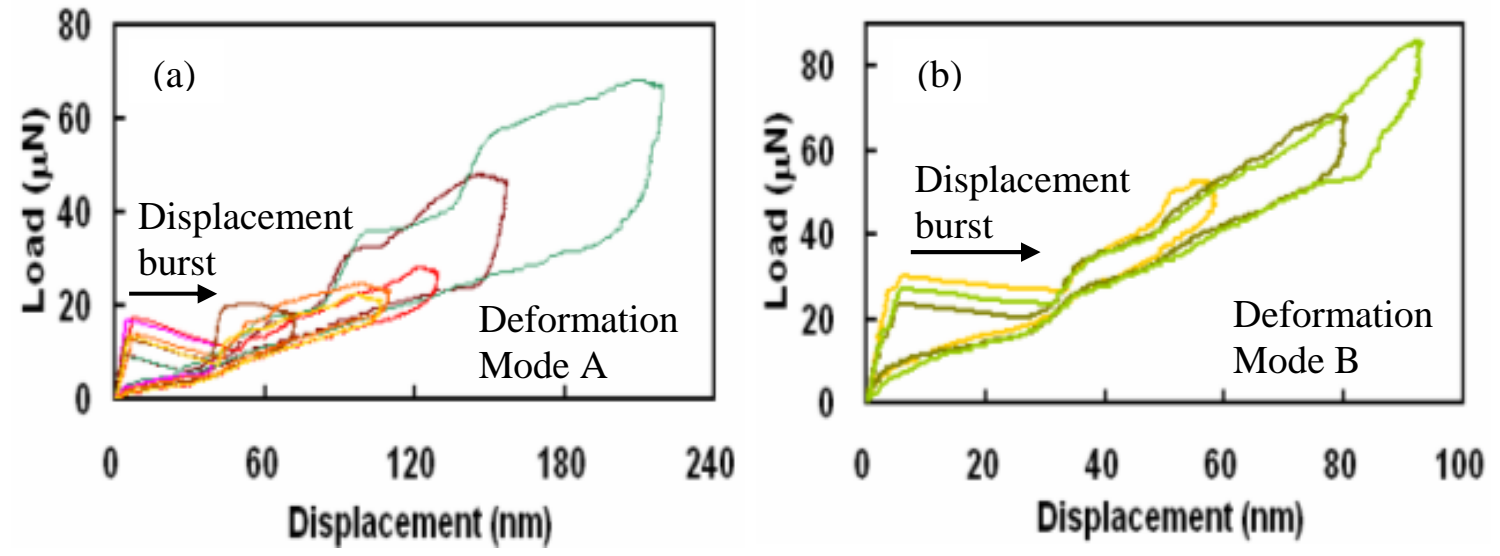

Fig.2. Load vs. displacement curves from the nanoindentation tests of the $24 \mathrm{~nm}$ SiNLs. (a) Deformation mode A, and (b) Deformation mode B. The SiNLs in (b) appeared to be stiffer than those in (a), with higher critical loads for the first displacement burst and smaller indentation displacements under same indentation forces. 


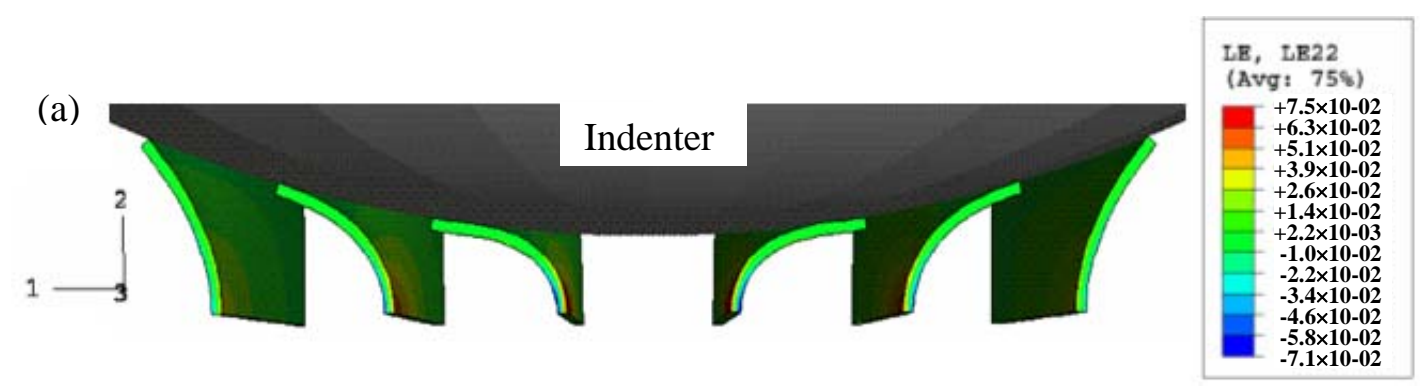

(b)

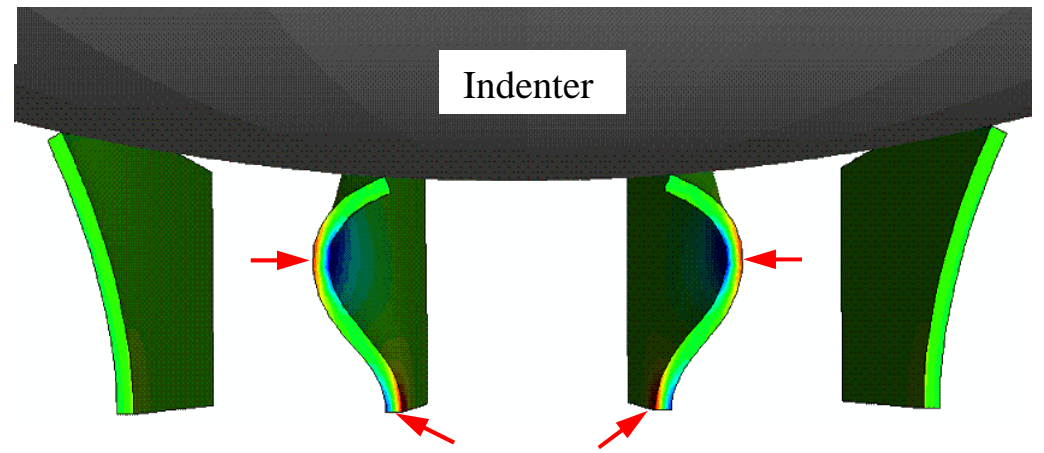

Fig 3. Finite element simulations of the $24 \mathrm{~nm}$ SiNLs under indenation. (a) Deformation mode A: The nanolines buckled with significant sliding at the contact with a low friction coefficient, $\mu<0.05$. The indentation displacement was $220 \mathrm{~nm}$. (b) Deformation mode B: The two center lines buckled with no sliding at the contact point for a higher friction coefficient $(\mu=0.06)$. The indentation displacement was $60 \mathrm{~nm}$ in this case. The red arrows indicate the locations of the maximum tensile stresses that may eventually cause fracture. 


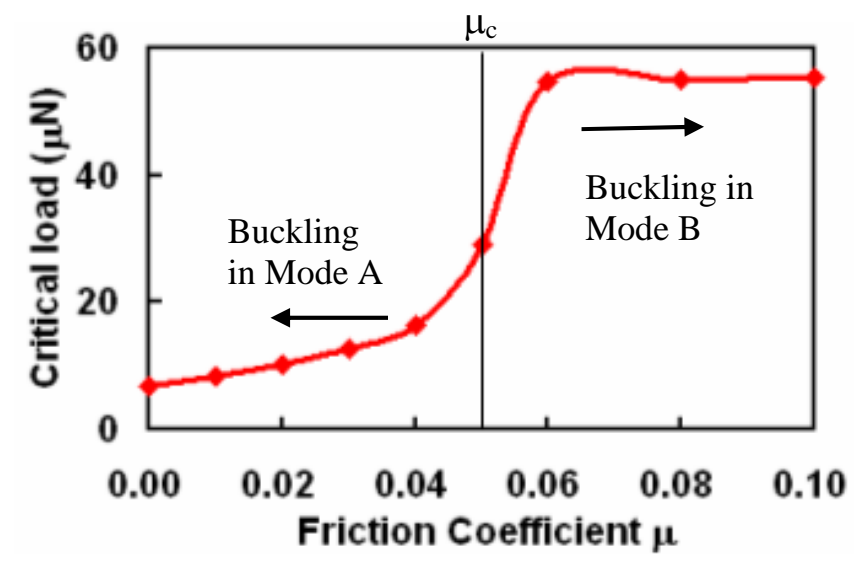

Fig. 4. The critical indentation load predicted by the FEM model, as a function of the friction coefficient between the indenter and the SiNLs. 


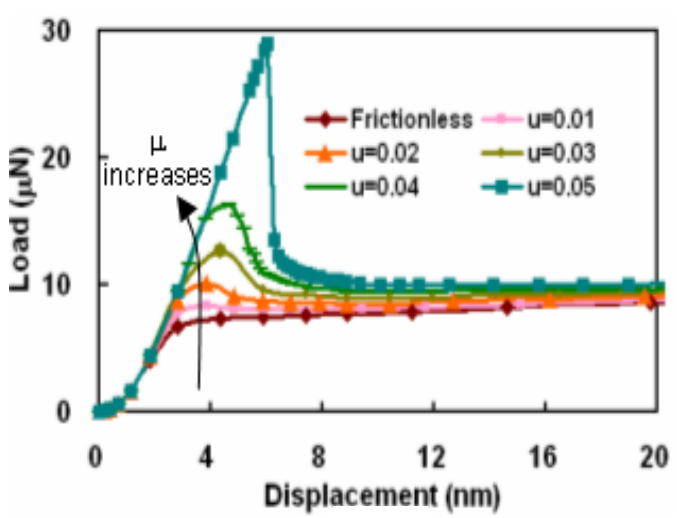

(a)

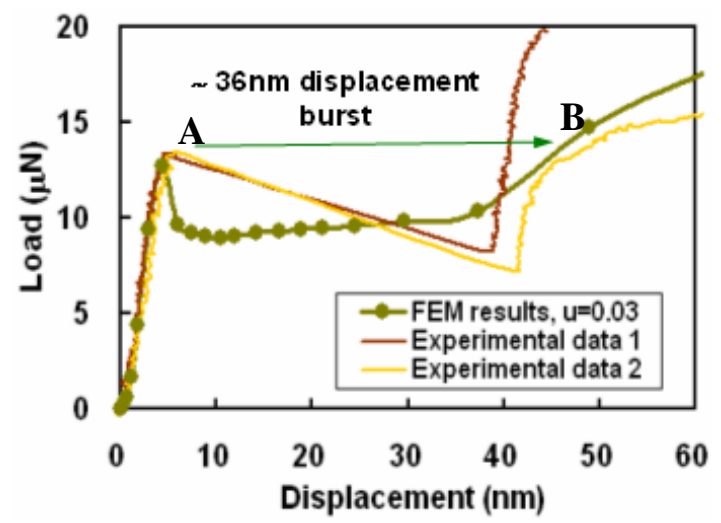

(c)

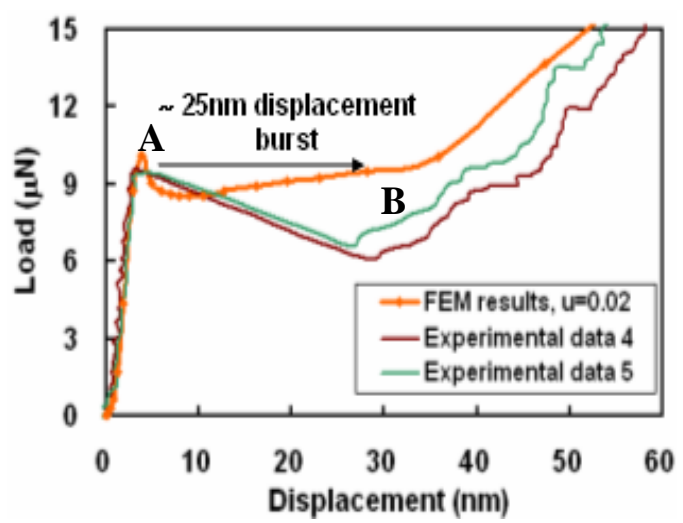

(b)

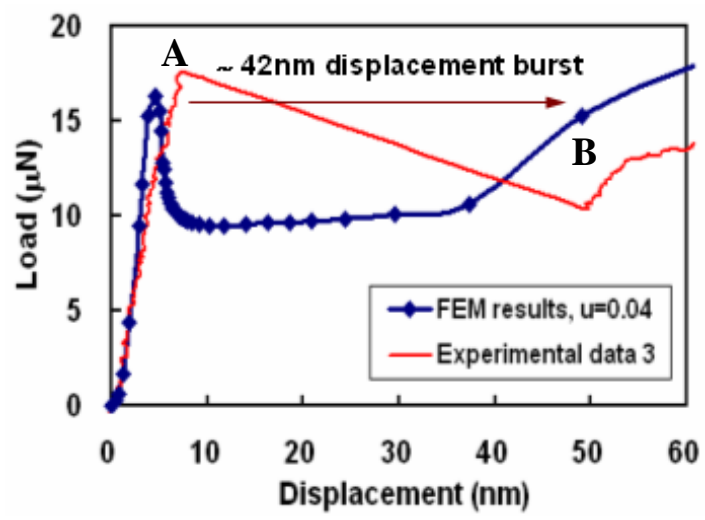

(d)

Fig. 5. Indentation force-displacement curves from finite element simulations of the $24 \mathrm{~nm}$ SiNLs. (a) Variation of the friction coefficient $\mu$ from 0 to 0.05 , showing increase of the critical load as $\mu$ increases. (b), (c) and (d): comparisons between the simulated load-displacement curves and the experimental data. The displacement bursts predicted by the FEM simulations are indicated by the horizontal arrows. 


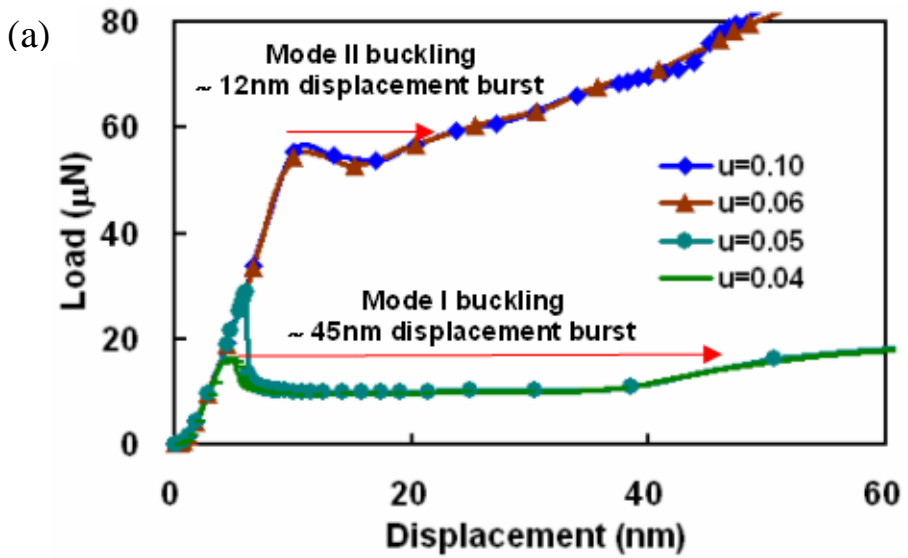

(b)

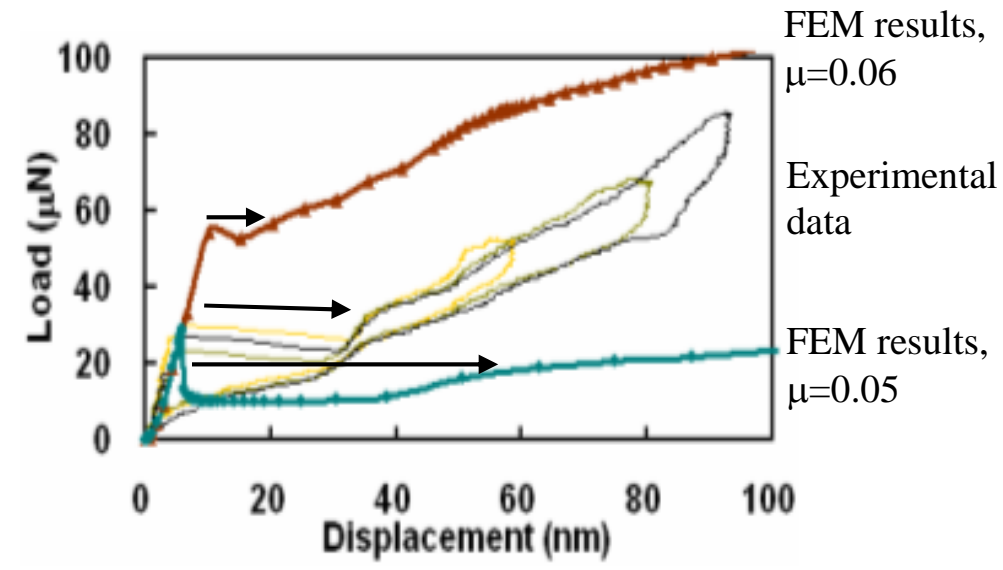

(c)

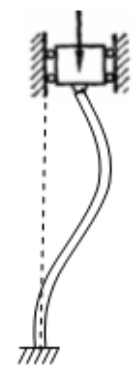

Fig. 6. (a) Simulated load vs. displacement curves of the $24 \mathrm{~nm}$ SiNLs under indentation with friction coefficient $\mu$ varying from 0.04 to 0.10 . (b) Comparison between the experimental data of deformation mode B (Fig. 2b) and the simulated load-displacement curves with friction coefficient $\mu=0.05$ and 0.06 . The magnitude 
of the displacement burst in each case was indicated by the horizontal arrows. (c) A schematic illustration of a hybrid buckling mode, with the top trapped after a partial sliding, possibly due to non-uniform friction properties at the contact surfaces. 

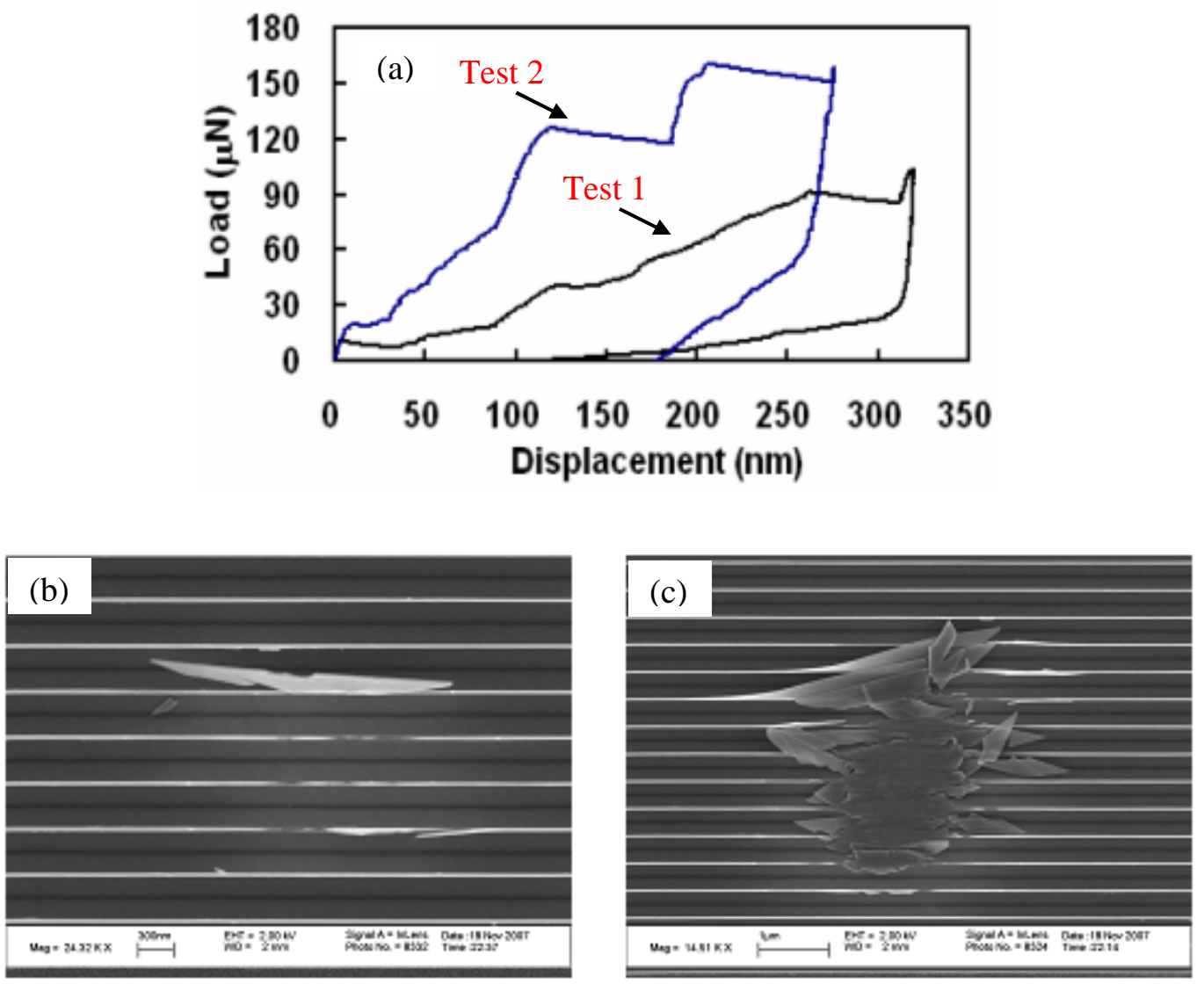

Fig. 7. Further increase of the indentation load far beyond the critical load resulted in fracture of SiNLs, with large residual displacements observed after unloading of the indenter. (a) Load-displacement curves from two indentation tests, which showed the characteristics of deformation modes A and B, respectively. (b) and (c) are two SEM images of the fractured SiNLs after Test 1 and Test 2, respectively. 


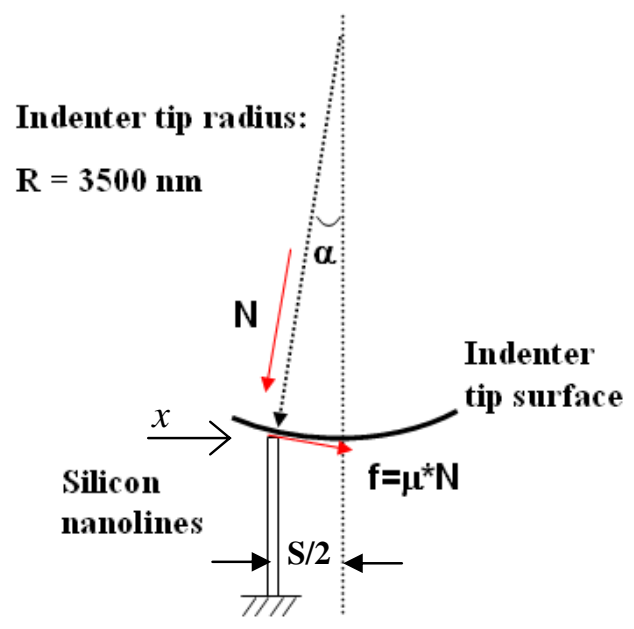

Fig. 8. Schematic of the contact geometry between the indenter and a nanoline, with the normal force $N$ and the friction force $f$ acting on the top of a nanoline. 